\title{
A review of quetiapine in combination with antidepressant therapy in patients with depression
}

\author{
Ella J Daly \\ Madhukar $\mathrm{H}$ Trivedi \\ Mood Disorders Program, \\ Department of Psychiatry, University \\ of Texas Southwestern Medical \\ School, Dallas, TX, USA
}

\begin{abstract}
Background: Atypical antipsychotics are increasingly used in the treatment of a broad spectrum of psychiatric disorders. There is evidence that in addition to treating the positive and negative symptoms of schizophrenia, as well as mania in bipolar disorder, these agents may have a potential role to play in the treatment of depressive disorders. In the following article we review the literature regarding the role of atypical antipsychotics, and specifically, quetiapine, in the treatment of major depressive disorder.
\end{abstract}

Materials and methods: In March 2007 the authors performed a Medline search (Englishlanguage) using the keywords quetiapine and depression, revealing a total of 47 articles published. We also looked for cross-references in the published articles, obtained data-on-file from AstraZeneca Pharmaceutical L.P., and included abstracts presented at conferences and recent meetings.

Results: From our review we found that there is increasing literature supporting the efficacy of add-on quetiapine in the treatment of major depressive disorder.

Conclusion: There is a need, however, for further well-designed, adequately powered, randomized, controlled trials to confirm this finding, specifically in unipolar depression.

Keywords: depression, adjunctive treatment, atypical antipsychotics, quetiapine

\section{Introduction}

Major depressive disorder (MDD) is a serious, debilitating illness that affects persons of all ages, races, and socioeconomic backgrounds. MDD occurs in up to one in eight individuals during their lifetime, making it one of the most prevalent of all medical illnesses. According to the Diagnostic and Statistical Manual-Fourth Edition Text Revision (DSM-IV TR) (American Psychiatric Association 2000), the point prevalence rates for MDD are approximately $2.3 \%-3.2 \%$ in men and 4.5\%-9.3\% in women, with a lifetime risk for developing an episode of $7 \%-12 \%$ for men and $20 \%-25 \%$ for women. Furthermore, depression currently ranks fourth for disability adjusted life years worldwide and is projected to be the second leading cause of disability worldwide by 2020 (World Health Organization 2001).

Unfortunately, although antidepressants are effective in the treatment of MDD, many patients do not achieve the desired goal of full remission (ie, absence of symptoms and return to full premorbid functioning) even with antidepressant trials at optimal doses and of adequate duration. The number of patients achieving symptom remission to initial antidepressant treatment is no more than $35 \%$ among all patients treated, with the remaining requiring at least two or more steps in pharmacotherapy (Rush et al 2004, 2006).

Treatment-resistant depression (TRD), depression that does not remit after one or more adequately delivered treatments (Souery et al 1999; Rush et al 2004), is a 
major and increasing public health burden due to its high prevalence, chronic and recurrent course, substantial morbidity, and significant direct and indirect costs (Greenberg et al 2003; Rush et al 2004, 2006). Patients with TRD are the most impaired and disabled of all patients with MDD (Murray and Lopez 1996; Greden 2001; Keller 2005), with psychosocial dysfunction contributing to treatment resistance (Papakostas et al 2004). Furthermore, treatment resistance may increase with increasing number of episodes, increasing episode duration, and particularly poor interepisodic recovery (Depression Guideline Panel 1993; Thase and Rush 1995). Treatment for this population needs to be aimed at producing full remission since anything short of remission is likely to result in relapse, recurrence, and future treatment resistance. The fact that $60 \%-70 \%$ of all patients with MDD meet criteria for TRD underscores the need for systematic development of innovative treatments for TRD (Insel 2006; Rubinow 2006). Currently, available clinical options after an initial optimal antidepressant trial (ie, at adequate doses and of sufficient duration) include switching to another antidepressant, as well as combination and augmentation strategies where another agent is added to the original antidepressant.

\section{Adjunctive antipsychotics in the treatment of major depressive disorder}

Phenothiazine antipsychotics have been shown to be effective in the treatment of nonpsychotic affective disorders (Schatzberg and Nemeroff 2006). However the high incidence of extrapyramidal side effects associated with these agents (especially in patients with mood disorder) has limited their use in this patient population. With the availability of the atypical antipsychotics, the risk of extrapyramidal side effects is now considerably reduced. Atypical antipsychotics have been shown to be an effective treatment for depressive symptoms associated with schizophrenia, bipolar disorder, posttraumatic stress disorder, and anxiety disorder in posthoc analyses from several studies (Tollefson et al 1998; Vieta et al 2001; Nemeroff et al 2002). In addition, clinical guidelines currently recommend atypical antipsychotics combined with antidepressants as first-line treatment for psychotic depression (Kennedy et al 2001).

In addition to widespread clinical use of atypical antipsychotics, there is now accumulating evidence that atypical antipsychotics may have a role to play in the treatment of patients with TRD (Hirschfeld et al 2002; Nemeroff 2005; Papakostas et al 2007). Preclinical studies have shown that the atypical antipsychotics risperidone and olanzapine have
$5-\mathrm{HT}_{2 \mathrm{~A}}$ antagonist effects, which may enhance the action of serotonin, and therefore augment the therapeutic effect of the selective serotonin reuptake inhibitors (SSRIs) (Ostroff and Nelson 1999; Pitchot and Ansseau 2001; Shelton et al 2001). In addition, animal studies have shown that the combination of SSRIs and atypical antipsychotics has synergistic effects on the release of dopamine and norepinephrine (Tarazi et al 2002). It has been suggested that prefrontal cortical activation may have salutary effects on mood (Hirschfeld et al 2002). Two atypical antipsychotics, clozapine and olanzapine, have been shown to increase dopamine release in the prefrontal cortex, whereas haloperidol (a conventional antipsychotic) does not (Tarazi et al 2002). Another atypical antipsychotic, risperidone, has also been found to have a more modest effect on norepinephrine and dopamine release in the prefrontal cortex.

Until recently, there had been few randomized controlled trials (RCTs) looking at the atypical antipsychotics as an augmentation strategy for TRD, with those that had been done presenting conflicting results (Papakostas et al 2007). However, there is preliminary evidence that risperidone may be effective as an adjunctive agent when combined with an antidepressant (O'Connor and Silver 1998; Ostroff and Nelson 1999; Hirose and Ashby 2002; Viner et al 2003; Nemeroff et al 2004). Similarly, there is also evidence from double-blind trials that augmentation of fluoxetine with olanzapine produces significantly greater improvement in depression compared with either olanzapine or fluoxetine monotherapy (Shelton et al 2001; Dube et al 2002). However, in two recently published large RCTs, by the end of the studies the authors found no significant difference between treatment with the fluoxetine and olanzapine combination (OFC) compared to either olanzapine or fluoxetine monotherapy, and nortriptyline (Shelton et al 2005) or venlafaxine (Corya et al 2006). It should be noted that in post hoc analysis conducted in the subgroup of patients who had failed an adequate selective serotonin reuptake inhibitor (SSRI) trial in the current major depressive episode, OFC showed a statistically significant difference at endpoint in one study compared to the olanzapine group (Shelton et al 2005) and in the second study (Corya et al 2006), a statistical difference was seen between the OFC and either olanzapine or fluoxetine monotherapy. Similarly, recently, Thase and colleagues (Thase et al 2007), found that OFC demonstrated significantly greater improvement in depressive symptoms compared olanzapine monotherapy in one of two studies and in a pooled analysis of patients with TRD. To date, other open label studies with both ziprasidone (Dunner et al 
2003; Papakostas et al 2004) and aripiprazole (Papakostas et al 2005; Simon and Nemeroff 2005) suggest both agents to be effective when used as adjunctive treatments to an antidepressant.

In a recent meta-analysis of 10 RCTs assessing adjunctive treatment of a standard antidepressant with an atypical antipsychotic agent (olanzapine, risperidone, or quetiapine), Papakostas et al found that patients randomized to adjunctive treatment with an atypical antipsychotic were more likely to experience remission or clinical response compared to those who received adjunctive placebo, with pooled remission and response rates for both groups $47.4 \%$ vs $22.3 \%$ and $57.2 \%$ vs $35.4 \%$, respectively (Papakostas et al 2007). Though requiring further investigation in terms of efficacy, safety, and tolerability as compared to other adjunctive strategies, all of the above data suggest that atypical antipsychotic augmentation of antidepressants may be a viable option for patients with TRD.

\section{Quetiapine}

\section{Pharmacological properties}

Quetiapine (Seroquel) was approved by the US Food and Drug Administration (FDA) in 1997 and is an atypical antipsychotic with established efficacy in the treatment of schizophrenia (Cheer and Wagstaff 2004). The preclinical profile of quetiapine is similar to the first atypical antipsychotic, clozapine, but with a reduced tendency to cause motor disturbances (Nemeroff et al 2002). Quetiapine is a dibenzothiazepine derivative for which the mechanism of action is unknown. It acts as an antagonist at serotonin 5-HT $\mathrm{HA}_{1 \mathrm{~A}}$ and 5- $\mathrm{HT}_{2 \mathrm{~A}}$, dopamine $\mathrm{D}_{1}$ and $\mathrm{D}_{2}$, histamine $\mathrm{H}_{1}$ and adrenergic $\alpha_{1}$ and $\alpha_{2}$ receptors (Dando and Keating 2005). Of note, it has a much higher level of occupancy of $5-\mathrm{HT}_{2 \mathrm{~A}}$ receptors compared to $\mathrm{D}_{2}$ receptors, a factor generally considered to be predictive of an atypical antipsychotic (Goldstein 1996). Quetiapine has negligible affinity for cholinergic muscarinic receptors, thereby contributing to its low risk for anticholinergic side effects. There is also evidence from animal models of low potential for extrapyramidal side effects (Cheer and Wagstaff 2004).

In terms of pharmacokinetics, the absorption of quetiapine is rapid, with the median time to maximum observed plasma concentration ranging from 1 to 2 hours (DeVane and Nemeroff 2001). Quetiapine is approximately $83 \%$ protein-bound, with a mean terminal half-life reported as approximately 7 hours. It is metabolized primarily in the liver, mainly by sulphoxidation by cytochrome P450 (CYP) 3A4 (Grimm et al 1997). Although, quetiapine neither induces nor inhibits CYP 3A4, caution is required when administering quetiapine with other drugs, which inhibit or induce the CYP 3A4 isoenzyme (DeVane and Nemeroff 2001). Quetiapine doses for the treatment of psychosis range from $150 \mathrm{mg}$ to $750 \mathrm{mg} /$ day.

\section{Quetiapine in the treatment of depressive symptoms in schizophrenia}

Mood disorders in patients with schizophrenia are associated with poor outcome, an increased risk of relapse, and a high rate of suicide (Meltzer et al 1998). It is estimated that the prevalence of syndromal depression in schizophrenia ranges from $25 \%$ to $60 \%$ (Martin et al 1985; Johnson 1988; Hirsch and Jolley 1989; Harrow et al 1994). There is evidence to date suggesting a positive effect associated with the atypical antipsychotics in patients with schizophrenia who experience depression (Muller-Siecheneder et al 1998; Tollefson et al 1998; Keck et al 2000). In terms of quetiapine, one group looked at data from two double blind, placebo-controlled, acute, 6-week trials evaluating the effects of quetiapine on depressive signs and symptoms in patients with schizophrenia (Arvanitis et al 1997). Data from both trials showed that quetiapine was superior to placebo in improving depressive symptoms, whereas haloperidol (used as a comparator) was not (Arvanitis et al 1997). Similarly, there is evidence supporting the maintenance of the efficacy of quetiapine in the treatment of depressive and anxiety symptoms in schizophrenia with long-term treatment (Kasper 2004).

\section{Quetiapine in the treatment of bipolar depression}

Bipolar depression is a recurrent and extremely debilitating phase of bipolar disorder, with patients with bipolar disorder overall experiencing more depressive symptoms than either mania or hypomania. The neurochemistry and pathogenesis of bipolar disorder remain poorly understood. In terms of treatment options, there is some evidence to date suggesting that treating bipolar depression with antidepressants alone may be associated with a possible increased risk of treatment-emergent mania (Grunze 2005). Currently lithium and lamotrigine are recommended as initial treatments for acute bipolar I depression (Hirschfeld et al 2002; Goodwin and Consensus Group of the British Association of Psychopharmacology 2003); however, the response rate rarely exceeds $50 \%$.

More recently, there is evidence of the efficacy of the atypical antipsychotics in the treatment of bipolar depression (Tohen et al 2003; Calabrese et al 2005). Like the olanzapine/ 
fluoxetine combination, quetiapine has recently been approved for this indication. The efficacy of oral quetiapine has been assessed in two recent positive, placebo-controlled trials demonstrating efficacy in the acute treatment of bipolar depression (Calabrese et al 2005; Thase et al 2006), as well as several other open-label trials (Sajatovic et al 2001; Milev et al 2006). Similarly, pooled data from the two placebocontrolled studies discussed above (Calabrese et al 2005; Thase et al 2006) show evidence supporting the efficacy of quetiapine monotherapy (either $300 \mathrm{mg}$ or $600 \mathrm{mg} /$ day) in patients with bipolar II disorder (Hirschfeld et al 2006).

Looking at the potential mechanism of action in bipolar depression, animal studies have shown that atypical antipsychotics such as quetiapine that block $5-\mathrm{HT}_{2 \mathrm{~A}}$ receptors down-regulate brain $5-\mathrm{HT}_{2 \mathrm{~A}}$ receptors (Tarazi et al 2002). It is suggested that down-regulation of $5 \mathrm{HT}_{2 \mathrm{~A}}$ seems to represent a strong point of commonality between quetiapine and antidepressant treatments (Yatham et al 2005). In addition, concomitant dampening by quetiapine of dopamine signaling in the mesolimbic pathways may be advantageous in preventing the dopamine-induced switching to hypomania that can occur with unimodal antidepressants (Yatham et al 2005).

\section{Quetiapine for major depression with psychotic features}

In terms of MDD with psychotic features, though there have been reports of quetiapine in the treatment of a variety of psychotic disorders (Zarate et al 2000; Sajatovic et al 2002), at the time of writing we are only aware of one 6-week, open label multi-center study looking at adjunctive quetiapine patients with unipolar depression with psychotic features (Konstantinidis et al 2007). This small study evaluated the efficacy of quetiapine in combination with citalopram in adults $(n=25)$ with DSM-IV diagnosis of unipolar psychotic depression. The primary endpoint assessed was change from baseline in HAM-D-21. The authors report that mean HAM-D was reduced from $31.21 \pm 5.18$ at baseline to $13.25 \pm$ 10.87 at week $6(p<0.05)$. In addition, significant improvement was also seen in psychotic symptoms as indicated by a decrease in the Brief Psychiatric Rating Scale (BPRS) from $59.25 \pm 6.60$ to $35.25 \pm 15.60$ at week $6(\mathrm{p}<0.001)$.

\section{Quetiapine as an adjunctive treatment for major depressive disorder}

As mentioned earlier, the fact that $60 \%-70 \%$ of all patients with MDD meet criteria for TRD underscores the need for systematic development of innovative treatments for TRD (Insel 2006; Rubinow 2006). While there is accumulating evidence supporting the strategy of antidepressant augmentation with atypical antipsychotics (Hirschfeld et al 2002; Nemeroff 2005; Papakostas et al 2007), the efficacy of this treatment strategy has not yet been firmly established. In view of the importance of further evaluating the potential role of atypicals in MDD and the large number of just completed trials, we will describe first the published literature for randomized controlled trials (RCTs), open label trials, as well as case series, and follow that up with the review conducted on poster presentations since a large number of RCTs are not yet published. We are only aware of two published randomized, controlled trials evaluating quetiapine in treatment-resistant depression (Yargic et al 2004; Hussain et al 2005), with further results from several other studies presented at recent meetings (Mattingly et al 2006; McIntyre et al 2006).

In their prospective, single-blind trial, Yargic and colleagues reported on patients with a DSM-IV diagnosis of MDD and associated anxiety who were randomly assigned to an 8-week treatment with paroxetine alone $(n=54)$ or paroxetine plus quetiapine $(n=58)$ (Yargic et al 2004). Quetiapine was given at a dose of up to $200 \mathrm{mg} / \mathrm{day}$ and paroxetine at a dose of up to $60 \mathrm{mg} / \mathrm{day}$. The authors found that decrease in HAM-D scores were significantly greater in the combined therapy group than with paroxetine alone throughout the study period $(\mathrm{p}<0.008)$. In addition, in terms of associated anxiety, the decrease in Hamilton Anxiety Rating Scale (HAM-A) scores was also significantly greater in the combined therapy group compared to paroxetine alone at weeks 2, 4, 6, and LOCF ( $\mathrm{p}<0.008$ ).

Similarly, in their study, Hussain and colleagues conducted a randomized, double-blind study in 72 patients with MDD comparing the efficacy of monotherapy antidepressant treatment with an SSRI or a Serotonergic Noradrenergic Reuptake Inhibitor (SNRI), compared to adjunctive quetiapine therapy in the treatment of MDD and in the maintenance of remission of symptoms (Hussain et al 2005). Patients were randomly assigned to one of four treatment groups: paroxetine monotherapy, venlafaxine monotherapy, paroxetine and quetiapine combination, or venlafaxine and quetiapine combination. Scores from the 17-item HAM-D were assessed at baseline, weeks 1, 3, 6, and 12, and every 6 months for 3 years. Improvement in and maintenance of HAM-D ${ }_{17}$ scores were seen in all four groups at week 3 and maintained at assessments over the 3-year study. Significant improvement in depressive symptoms and the development of remission occurred more frequently in the paroxetine and quetiapine 
combination group. However, a greater overall frequency of adverse events was also observed in the combination therapy groups vs the monotherapy groups.

There is also preliminary evidence from open-label studies (Vavrusova 2002; Adson et al 2004; Sagud et al 2006; Baune et al 2007; Doree et al 2007) and case reports (Pathak et al 2005; Devarajan et al 2006) supporting the potential benefits of quetiapine in this area (see Table 1). In a recent publication, Doree and colleagues report on an 8-week, open-label, pilot study in which they compared the effects of quetiapine and lithium when used as an adjunct to antidepressant medication in 20 patients with MDD (Doree et al 2007). Prior to commencing adjunctive treatment with either agent, participants had a HAM-D score $\geq 20$ after 4 weeks at maximal antidepressant dose. Lithium was initiated at a dose of $600 \mathrm{mg} /$ day and adjusted in time to achieve blood levels between $0.8-1.2 \mathrm{nmol} / \mathrm{L}$. Quetiapine was titrated to a maximum of $400 \mathrm{mg} /$ day in the first week and then to a maximum of $800 \mathrm{mg} /$ day as clinically indicated. Outcome measures included difference in HAM-D and Montgomery Asberg Depression Rating Scale (MADRS) from baseline to week 8. Data from 20 patients were analyzed (10 on lithium, 10 on quetiapine). Results showed that depression, measured by the HAM-D significantly improved from baseline in both quetiapine $\left(\mathrm{F}_{1,90}=25.11, \mathrm{p}<0.0001\right)$ and lithium $\left(\mathrm{F}_{1,90}=34.54\right.$, $\mathrm{p}<0.0001)$, with a difference in improvement between the two groups (in favor of quetiapine) seen at day 14, and present at all time points thereafter $(\mathrm{p}<0.05)$. Similarly, significant improvement was seen on the MADRS in both the quetiapine group $\left(\mathrm{F}_{1,90}=68.89, \mathrm{p}<0.0001\right)$ and lithium $\left(\mathrm{F}_{1,90}=35.11\right.$, $\mathrm{p}<0.0001)$, with a significant difference in improvement in favor of quetiapine seen at week 4 ( $\mathrm{p}<0.05$ ). Evaluation on the HAM-D showed a response rate of $80 \%$ and $50 \%$ for quetiapine and lithium respectively, with a remission rate of $80 \%$ and $40 \%$. Similarly, on the MADRS the response rate was $80 \%$ and $50 \%$, and the remission rate was $80 \%$ and $30 \%$ for quetiapine and lithium respectively (see Table 1).

In another recently published, small, open-label, noncomparative, flexible-dosed, 20-week study evaluated the effects of quetiapine as an add-on therapy in patients with TRD who were refractory to previous treatments (Sagud et al 2006). The mean dose of quetiapine during the 20 -week trial was $515 \pm 109 \mathrm{mg} /$ day. Patients were evaluated at different points in time throughout the study with the HAM-D rating scale. Of the 18 patients enrolled in the study at baseline, 14 patients completed the open-label study. After the fourth week of treatment, results showed that augmentation with quetiapine significantly reduced HAM-D total scores, as well as scores on the HAM-A anxiety subscale. After the fifth week of treatment, scores on the HAM-D depressed mood subscale were also significantly reduced. Quetiapine addon therapy was also associated with a significant decrease in the HAM-D insomnia subscale after the second week of treatment (Sagud et al 2006).

In an earlier open-label pilot study, Vavrusova also reported on a trial of adjunctive quetiapine $(n=13)$ or adjunctive haloperidol $(n=13)$ in patients with severe, nonpsychotic depression not responding to 4 weeks of treatment with citalopram (Vavrusova 2002). Outcome measures included the HAM-D, the HAM-A, and the Positive and Negative Syndrome Scale (PANSS). At a dose of $200 \mathrm{mg} /$ day, quetiapine was found to significantly improve levels of depression, suicidal ideation, and retardation, as well as improving anxiety and sleep compared to haloperidol.

Another recently published 4-week open label study looked at clinical outcomes, quality of sleep and daytime motor activity in patients with treatment resistant major depression $(n=21)$ or bipolar depression $(n=6)$ who received a standard antidepressant (venlafaxine, escitalopram) plus a flexible dose of quetiapine (Baune et al 2007). Daily doses of quetiapine ranged between $300 \mathrm{mg} /$ day up to a maximum of $800 \mathrm{mg} /$ day over the 4 week study. Outcome measures included the HAM-D-21, the Pittsburgh Sleep Quality Index (PSQI), with motor activity continuously measured by actigraphy. At admission, the HAM-D-21 score was 20.2 (SD \pm 6.8 ), and after treatment it was 9.4 (SD \pm $4.8)$, and $4.9(\mathrm{SD} \pm 3.2)$ at discharge $(\mathrm{p}<0.001)$. It should be noted that all open label studies cited above were of small sample size and lacked a control group.

Further evidence suggesting the potential of quetiapine as an augmentation agent for TRD comes from a recent naturalistic case series, in which the authors present outcomes for six consecutive patients with nonpsychotic TRD - treated with the combination of sertraline (SSRI) and quetiapine (Devarajan et al 2006). All patients initially received sertraline monotherapy and then commenced adjunctive quetiapine after 6 or 8 weeks because of lack of significant clinical effect. Results showed that sertraline had minimal effect on baseline 21-item HAM-D scores; however, adding quetiapine improved ratings and outcomes at week 5-6 (Devarajan et al 2006). The authors suggest that the effectiveness of the combination of the SSRI antidepressant and quetiapine may result from the targeting of multiple neurotransmitters/receptors and specific mood-modulating neuroanatomical regions of interest. Additionally, they speculate that improvement in quality of sleep may have contributed to the improved 


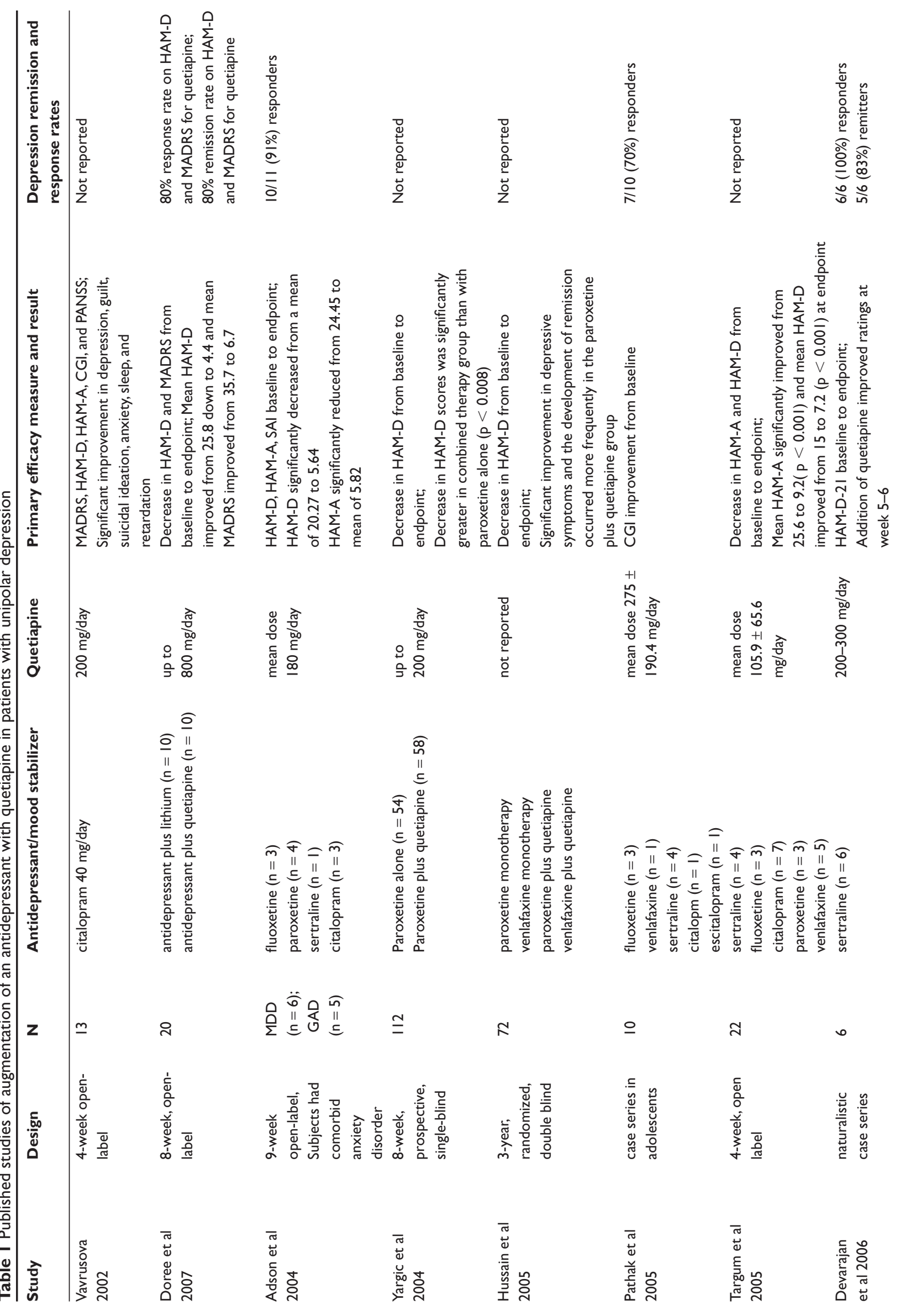


mental well-being of patients treated with add-on quetiapine (Devarajan et al 2006).

There is also preliminary evidence for use of quetiapine as an adjunctive treatment for treatment-resistant adolescent MDD (Pathak et al 2005). In a recently published case series, Pathak et al report on the use of adjunctive quetiapine in 10 adolescents with MDD who had failed to respond to a prior trial of SSRI of at least 8 weeks duration (Pathak et al 2005). Seven of 10 subjects (70\%) showed much or very much improved on clinical global improvement (CGI) rating scale (ie, score 1 or 2). The median dose of quetiapine prescribed was $200 \mathrm{mg}$ (mean $\pm \mathrm{SD}=275 \pm 190.4 \mathrm{mg}$; range 150-800 mg). Side effects included sedation (40\%) and weight gain (mean $\pm \mathrm{SD}=4.5 \pm 7.24$ pounds). The authors noted that the associated weight gain seen with quetiapine in this case series should be taken into consideration while calculating the risk-benefit ratio in the management of treatment-resistant depression in this patient population. No serious adverse events were reported.

In addition to the studies and case reports cited above, there are currently eight double blind, placebo-controlled studies ongoing at present looking at quetiapine either as a monotherapy or as an augmentation agent in the treatment of major depressive disorder (see http://www.clinicaltrials. gov).

\section{Randomized trials of quetiapine presented as posters}

In a recent poster presentation, McIntryre and colleagues presented data from a double-blind, randomized, placebocontrolled study in 58 patients, which evaluated the use of quetiapine as an adjunctive treatment to either SSRIs or SNRIs for patients with MDD associated with residual depressive and prominent anxiety symptoms (McIntyre et al 2006). Prior to receiving adjunctive treatment, study participants had received at least 6 weeks of SSRI/SNRI treatment, and were then randomly assigned to receive adjunctive quetiapine $(n=29)$ or adjunctive placebo $(n=29)$. Quetiapine was gradually titrated up to a maximum of $600 \mathrm{mg}$ /day as clinically indicated. The mean dose of quetiapine was $182 \pm$ $69 \mathrm{mg} /$ day. The primary outcome was mean change from baseline to week 8 in HAM-D and HAM-A scores (last observation carried forward [LOCF] analysis). Results: 18/29 quetiapine-treated patients and 16/29 placebo-treated patients completed the study. Significant improvement from baseline was seen in the HAM-D score at week 1 ( $-6.5 \mathrm{vs}-2.9$; $\mathrm{p} \leq 0.01)$ and at week $8(-11.2 \mathrm{vs}-5.5 ; \mathrm{p} \leq 0.01)$ in the quetiapine vs placebo group, respectively. HAM-D response rates 
(48\% vs $28 \%$ ) and remission rates (31\% vs $17 \%$ ) were also higher in the quetiapine group compared to placebo.

Similarly, Mattingly et al recently presented a poster documenting their findings from an 8-week, double-blind, randomized, placebo-controlled trial evaluating the efficacy of adjunctive quetiapine in depressed patients who were partial responders to at least 6 weeks of SSRI/SNRI treatment (Mattingly et al 2006). In this study patients with a baseline HAM-D $\geq 20$ were randomly assigned to receive either adjunctive quetiapine $200-400 \mathrm{mg} /$ day $(\mathrm{n}=23)$ or placebo $(n=13)$, with randomization occurring in a $2: 1$ ratio in favor of quetiapine. The primary outcome measure was change in HAM-D score from baseline to week 8 . The investigators found that HAM-D scores significantly improved from baseline (25.0 vs 24.5$)$ to endpoint (8.3 vs 14.7$)$ in the quetiapine vs placebo groups, respectively $(\mathrm{p}<0.01)$. Similarly, MADRS scores improved from baseline (32.4 vs. 33.5 ) to endpoint (15.4 vs 24.8) in the quetiapine vs. placebo groups, respectively ( $\mathrm{p}<0.05$ ). Of note, the authors report that they used a paired t-test and LOCF as the statistical approach for this analysis. Specifically, more patients were responders $(67 \%$ vs $27 \%, \mathrm{p}=0.015)$ or remitters $(43 \%$ vs $15 \%, \mathrm{p}<0.05)$ in the quetiapine group compared to the placebo group (see Table 2).

Finally, in yet another poster presentation, Khullar et al present data from a small, double blind trial comparing adjunctive quetiapine to placebo in 16 patients who had failed a 6-week trial of adequate doses of an SSRI or SNRI (Khullar et al 2006). The average dose of quetiapine used was $350 \mathrm{mg} /$ day. An LOCF analysis using independent samples t-tests showed significantly greater $(\mathrm{p}<0.05)$ mean changes in the HAM-D ${ }_{17}$, MADRS, and HAM-A for the quetiapine group $(n=8)$ versus the placebo group $(n=7)$. One limitation of this study is the small sample size.

\section{Quetiapine as a treatment for anxiety symptoms associated with MDD}

One recent 4-week, open label study evaluated the anxiolytic, antidepressive, and sleep effects and safety of quetiapine in patients with MDD who were on stable doses of SSRIs and presented with persistent anxiety (Targum et al 2005). Prior to entry into the study, eligible patients had been taking either an SSRI or SNRI for at least 6 weeks, and had a HAM-A $\geq 20$ and a HAM-D $\leq 17$ at screening and baseline. A total of 17 patients completed the study. Mean quetiapine doses achieved were $105 \pm 65.6 \mathrm{mg} /$ day. Results at endpoint showed significant improvement in HAM-A scores $(25.6 \pm$ 5.5 baseline to $9.2 \pm 5.5) ; \mathrm{p}<0.001)$. The HAM-D score also improved from $15 \pm 1.8$ at baseline to $7.2 \pm 5.0$ at endpoint $(\mathrm{p}<0.001)$.

Similarly, in their 9-week, open-label, flexible-dosed study, Adson and colleagues assessed quetiapine as an adjunctive therapy for SSRIs in 11 patients with either anxiety symptoms complicating a depressive disorder or an anxiety disorder (Adson et al 2004). Of the 11 patients participating in the study, 6 had a diagnosis of MDD with a comorbid anxiety disorder. To be eligible for the 9-week, open-label, flexible-dosed study, patients had to be currently treated with an SSRI at an adequate dose for at least 6 weeks. Quetiapine was titrated gradually based on tolerability and effect during the first 3 weeks up to a maximum total daily dose of $300 \mathrm{mg}$ (100 mg qam and $200 \mathrm{mg}$ qhs). A total of 10 patients completed the study. Clinically relevant reductions in mean HAM-D, HAM-A, and the State Anxiety Index (SAI) scores from baseline were seen as early as week 1 , with sustained improvement in symptoms on all three measures over the 9-week period. HAM-D scores decreased from a mean of 20.27 to 5.64 at the final visit, while HAM-A scores decreased from a mean of 24.45 at baseline to 5.82 at study completion (Adson et al 2004) (see Table 1).

There is also evidence from case series supporting quetiapine as a monotherapy in MDD and comorbid anxiety disorder. In one case series of 36 patients with a DSM-IV diagnosis of generalized anxiety disorder (GAD) (22 patients) or GAD with panic disorder (14 patients), 27 suffered from comorbid MDD (Galynker et al 2005). Patients were treated with either risperidone $(n=23)$ or quetiapine $(n=$ 13). Risperidone was commenced at a total daily dose of $0.125 \mathrm{mg} /$ day and gradually titrated to a maximum dose of $0.5 \mathrm{mg} /$ day. Patients on quetiapine started on a total daily dose of $25 \mathrm{mg} /$ day and gradually were titrated to a maximum of $300 \mathrm{mg} /$ day. Outcomes were assessed with the HAM-A and modified HAM-D (with two anxiety items removed). A total of 32 patients completed the study. Mean baseline HAM-D scores were $24.95 \pm 10.01$ and $20.31 \pm 7.79$ for patients receiving risperidone or quetiapine, respectively. Mean posttreatment HAM-D scores were $6.37 \pm 4.96(\mathrm{p}<$ $0.001)$ and $12.15 \pm 5.44(\mathrm{p}<0.001)$ for patients treated with risperidone or quetiapine, respectively. Mean baseline HAM-A scores were $21.42 \pm 5.53$ for patients treated with risperidone and $24.92 \pm 6.45$ for patients treated with quetiapine. Mean posttreatment HAM-A scores were $5.68 \pm 5.03$ for patients treated with risperidone and $7.46 \pm 5.71$ for patients treated with quetiapine. Specifically, of the 13 patients taking quetiapine, 10 (77\%) demonstrated an improvement in HAM-A scores by at least 50\%, with 4 (31\%) demonstrating 


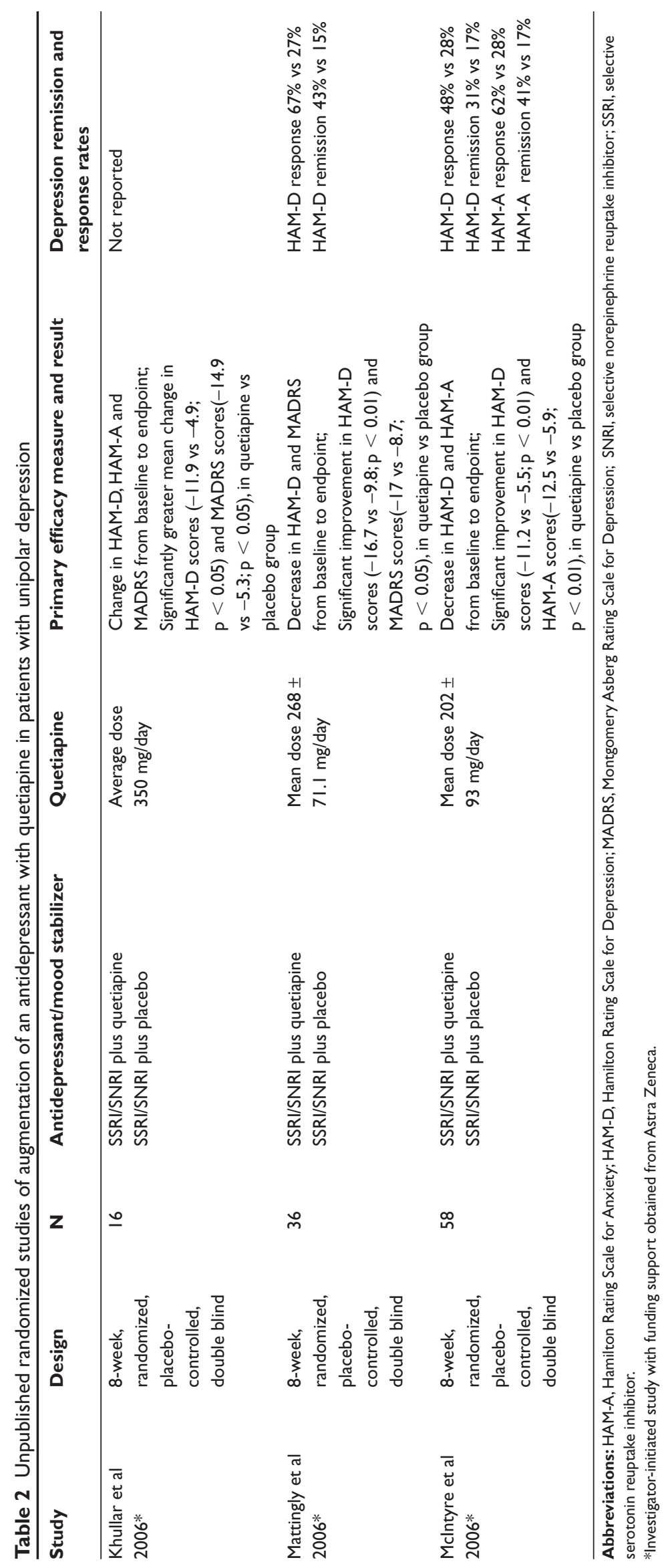


improvement in HAM-D scores by at least 50\% (Galynker et al 2005). The mean daily dosages of risperidone and quetiapine were $0.21 \pm 0.11 \mathrm{mg}$ and $105.8 \pm \mathrm{mg}$, respectively.

\section{Tolerability and safety profile}

Studies to date (mainly in schizophrenia) have demonstrated a low propensity of quetiapine to produce extrapyramidal side effects (EPS) or elevated prolactin levels (Kapur et al 2002; Lieberman and Perkins 2002). Extrapyramidal side effects include akathisia, dystonia, and Parkinsonism. In vivo functional studies with quetiapine all provide evidence that quetiapine has a preferential effect on limbic as opposed to striatal $D_{2}$ receptors (Nemeroff et al 2002). Extrapyramidal side effects are associated with $\mathrm{D}_{2}$ occupancy in the striatum, therefore predicting low levels of EPS for quetiapine. Similarly, unlike olanzapine and risperidone, the lack of effect of quetiapine on dopamine receptors in vitro studies in the basal ganglia provides further evidence to suggest low levels of EPS with quetiapine (Tarazi et al 2001). Finally, it has been suggested that because quetiapine is loosely bound to $\mathrm{D}_{2}$ receptors, its rapid release from $\mathrm{D}_{2}$ receptors may contribute to their low $\mathrm{D}_{2}$ occupancy and lower potential to cause EPS (Seeman and Tallerico 1998).

Prolactin elevation appears to be associated with the blockade of $\mathrm{D}_{2}$ receptors at the level of the anterior pituitary lactotrophs, where dopamine exerts an inhibitory effect on prolactin secretion (Jaber et al 1996). Unlike the striatum, the anterior pituitary lies outside the blood-brain barrier. In terms of atypical antipsychotics, compounds displaying a higher peripheral potency have been shown to bring about higher prolactin levels for a given level of functional central antagonism (Kapur et al 2002). In contrast, quetiapine has been shown in animal studies to have a relatively low differential occupancy of $\mathrm{D}_{2}$ receptors in the striatum compared versus the pituitary, thereby further explaining its low propensity to cause hyperprolactinemia (Kapur et al 2002).

Most of the existing data regarding the safety and tolerability of quetiapine relates to its use in patients with schizophrenia, however there is preliminary data emerging from studies looking at quetiapine in affective disorders. Data concerning tolerability of quetiapine in patients with bipolar depression is now available from the BOLDER I and II trials (Calabrese et al 2005; Thase et al 2006), with evidence that quetiapine is generally well tolerated in patients with bipolar depression (Keating and Robinson 2007). Across both studies EPS was reported in 6.7\% and $12.3 \%$ of those receiving quetiapine $300 \mathrm{mg} /$ day, $8.9 \%$ and $10.1 \%$ of those receiving the $600 \mathrm{mg}$ /day dose, and $2.2 \%$ and $6.6 \%$ of those receiving placebo (Calabrese et al 2005; Thase et al 2006). No clinically significant differences were seen across treatment groups in terms of vital signs, ECG readings, or laboratory parameters. Of note, there was no significant difference in the incidence of treatment-emergent mania between quetiapine and placebo in either study.

Another factor to evaluate when considering using adjunctive quetiapine for MDD is the issue of weight gain. In a recent review article, Gentile reviews the risk of weight gain associated with long-term treatment with atypical antipsychotics (Gentile 2006). Previous analyses of weight changes in patients participating in trials of quetiapine (controlled, uncontrolled, and open label-extensions) suggest that the risk for quetiapine-induced weight-gain is not dose-related. According to Gentile's review, however, there is conflicting data regarding weight gain associated with quetiapine, with some studies showing only modest weight gain (Kasper and Muller-Spahn 2000; Brecher et al 2004; Nagy 2005), and others reporting clinically relevant weight gain (McIntyre et al 2003; Sprague et al 2004). The author reports that in shortterm studies, a definite rank order of weight-gain potential among atypical antipsychotic has been demonstrated with clozapine related to the highest risk of weight gain, followed in decreasing order of magnitude by olanzapine, quetiapine, risperidone, amisulpride, aripiprazole, and ziprasidone. In contrast, in long-term studies, apart from clozapine at one end of the spectrum and ziprasidone at the other, the difference in weight gain shown by the other atypical antipsychotics became less intense (Gentile 2006).

The recent BOLDER I and II studies report a mean weight gain of 1.0 and $1.4 \mathrm{~kg}$ with quetiapine $300 \mathrm{mg} / \mathrm{day}$, 1.6 and $1.3 \mathrm{~kg}$ with the $600 \mathrm{mg}$ /day dose, compared to 0.2 and $0.3 \mathrm{~kg}$ with placebo (Calabrese et al 2005; Thase et al 2006). To date there is little information available in terms of major depressive disorder, however, a recent study cited earlier, looking at MDD with psychotic features reported an average weight change of $+2.1 \mathrm{~kg}( \pm \mathrm{SD})$, with mean weight at visit $1,72.72( \pm 16.34) \mathrm{kg}$ and mean weight at visit $4,74.79$ $( \pm 18.69) \mathrm{kg}$ (Konstantinidis et al 2007).

\section{Discussion}

Treatment-resistant major depressive disorder is a common problem in clinical practice and often poses a considerable challenge to the treating physician. Prior studies suggest that atypical antipsychotics have a role to play in the treatment of treatment-resistant MDD (Hirschfeld et al 2002). From our review of the literature above, we found growing evidence specifically supporting the use of quetiapine as an 
adjunctive agent in patients with TRD, particularly in patients with residual symptoms of anxiety and sleep difficulties. Unfortunately, few randomized controlled trials are available through peer-reviewed publications.

As an atypical antipsychotic with a low propensity to cause either extrapyramidal side effects or hyperprolactinemia, quetiapine has been shown to be a potential treatment in a broad range of psychiatric disorders (Adityanjee and Schulz 2002). Specifically, in terms of depressive symptoms, we see that there is accumulating evidence supporting the benefits of quetiapine as an adjunctive treatment in TRD as well as bipolar depression. Even though the exact mechanism of action is not known, it appears to relate to the $5-\mathrm{HT}_{2 \mathrm{~A}}$ antagonist effects, which may enhance the action of serotonin, and therefore augment the therapeutic effect of the selective serotonin reuptake inhibitors (SSRIs). Similarly, animal studies have shown that the combination of SSRIs and atypical antipsychotics has synergistic effects on the release of dopamine and norepinephrine (Tarazi et al 2002).

To date, there are only a few randomized trials looking at adjunctive quetiapine in the treatment of major depressive disorder. Given the prevalence of treatment resistance in this disorder, as well as the evidence from studies to date, further placebo-controlled trials are indicated at this time. In addition to efficacy, the potential for weight gain associated with the use of atypical antipsychotics is also an issue for clinicians to consider in their clinical decision-making. Overall, however, preliminary evidence suggests that quetiapine may be an effective treatment to be added to our current armamentarium of options for this patient population.

\section{Disclosures}

Dr. Trivedi has been a consultant for Abbott Laboratories, Inc.; Akzo (Organon Pharmaceuticals Inc.); AstraZeneca; Bayer; Bristol-Myers Squibb Company; Cephalon, Inc.; Cyberonics, Inc.; Eli Lilly and Company; Fabre-Kramer Pharmaceuticals, Inc. Forest Pharmaceuticals; GlaxoSmithKline; Janssen Pharmaceutica Products, LP; Johnson and Johnson PRD; Eli Lilly and Company; Meade Johnson; Neuronetics; Parke-Davis Pharmaceuticals, Inc.; Pfizer, Inc.; Pharmacia and Upjohn; Sepracor; Solvay Pharmaceuticals, Inc.; VantagePoint; Wyeth-Ayerst Laboratories. He has served on speakers bureaus for Abdi Brahim; Akzo (Organon Pharmaceuticals Inc.); Bristol-Myers Squibb Company; Cephalon, Inc.; Cyberonics, Inc.; Forest Pharmaceuticals; GlaxoSmithKline; Janssen Pharmaceutica Products, LP; Eli Lilly and Company; Pharmacia and Upjohn; Solvay Pharmaceuticals, Inc.; Wyeth-Ayerst Laboratories. He has also received grant support from Bristol-Myers Squibb Company; Cephalon, Inc.; Corcept Therapeutics, Inc.; Cyberonics, Inc.; Eli Lilly and Company; Forest Pharmaceuticals; GlaxoSmithKline; Janssen Pharmaceutica; Merck; National Institute of Mental Health; National Alliance for Research in Schizophrenia and Depression; Novartis; Pfizer Inc.; Pharmacia and Upjohn; Predix Pharmaceuticals; Solvay Pharmaceuticals, Inc.; Wyeth-Ayerst Laboratories. Dr. Daly has no disclosures to report.

\section{References}

Adityanjee, Schulz SC. 2002. Clinical use of quetiapine in disease states other than schizophrenia. J Clin Psychiatry, 63:32-8.

Adson DE, Kushner MG, Eiben MK, et al. 2004. Preliminary experience with adjunctive quetiapine in patients receiving selective serotonin reuptake inhibitors. Depress Anxiety, 19:121-6.

American Psychiatric Association. 2000. Diagnostic and statistical manual of mental disorders - text revision. Washington, DC.

Arvanitis LA, Miller BG, Borison RL, et al. 1997. Multiple fixed doses of "Seroquel" (quetiapine) in patients with acute exacerbation of schizophrenia: a comparison with haloperidol and placebo. Biol Psychiatry, 42:233-46.

Baune BT, Caliskan S, Todder D. 2007. Effects of adjunctive antidepressant therapy with quetiapine on clinical outcome, quality of sleep and daytime motor activity in patients with treatment-resistant depression. Hum Psychopharmacol, 22:1-9.

Brecher M, Zukin S, Leong R, et al. Long-term weight change with quetiapine treatment in schizophrenia: a comprehensive data review [poster] American College of Neuropsychopharmacology 43rd Annual Meeting. San Juan; 2004.

Calabrese JR, Keck PE, Macfadden W, et al. 2005. A randomized, doubleblind, placebo-controlled trial of quetiapine in the treatment of bipolar I or II depression. Am J Psychiatry, 162:1351-60.

Cheer SM, Wagstaff AJ. 2004. Quetiapine - A review of its use in the management of schizophrenia. CNS Drugs, 18:173-99.

Corya SA, Williamson D, Sanger TM, et al. 2006. A randomized, doubleblind comparison of olanzapine/fluoxetine combination, olanzapine, fluoxetine, and venlafaxine in treatment-resistant depression. Depress Anxiety, 23:364-72.

Dando TM, Keating GM. 2005. Quetiapine - A review of its use in acute mania and depression associated with bipolar disorder. Drugs, 65:2533-51.

Depression Guideline Panel. 1993. Clinical practice guideline. depression in primary care: volume 2: treatment of major depression. Publication no. 93-0551.: U.S. Department of Health and Human Services - Agency for Health Care Policy and Research.

DeVane CL, Nemeroff CB. 2001. Clinical pharmacokinetics of quetiapine An atypical antipsychotic. Clin Pharmacokinet, 40:509-22.

Devarajan S, Ali J, Dursun SM. 2006. Quetiapine plus SSRI in treatmentresistant depression: possible mechanisms. Psychopharmacology, 185:402-3.

Doree JP, Des RJ, Lew V, et al. 2007. Quetiapine augmentation of treatment-resistant depression: a comparison with lithium. Curr Med Res Opin, 23:333-41.

Dube S, Andersen SW, Paul S, et al. 2002. Metanalysis of olanzapinefluoxetine use in treatment-resistant depression. Eur Neuropsychopharmacol, 12:S180.

Dunner D, Amsterdam J, Shelton R, et al. 2003. Adjunctive ziprasidone in treatment-resistant depression: A pilot study (Abstract NR238). The 156th APA Annual Meeting, San Francisco, CA.

Galynker I, Khan A, Grebchenko Y, et al. 2005. Low-dose risperidone and quetiapine as monotherapy for comorbid anxiety and depression. J Clin Psychiatry, 66:544. 
Gentile S. 2006. Long-term treatment with atypical antipsychotics and the risk of weight gain - A literature analysis. Drug Saf, 29:303-19.

Goldstein J. 1996. Preclinical profile of seroquel (quetiapine): an atypical antipsychotic with clozapine-like pharmacology. In: Schizophrenia: breaking down the barriers. New York, NY: Wiley and Sons.

Goodwin GM, Consensus Group of the British Association of Psychopharmacology. 2003. Evidence-based guidelines for treating bipolar disorder: recommendations from the British Association for Psychopharmacology. J Psychopharmacol, 17:149-73.

Greden JF. 2001. The burden of disease for treatment-resistant depression. $J$ Clin Psychiatry, 62:S26-31.

Greenberg PE, Kessler RC, Birnbaum HG, et al. 2003. The economic burden of depression in the United States: how did it change between 1990 and 2000? J Clin Psychiatry, 64:1465-75.

Grimm SW, Stams KR, Bui K. 1997. In vitro prediction of potential metabolic drug interactions for Seroquel. Schizophr Res, 24:198.

Grunze H. 2005. Reevaluating therapies for bipolar depression. J Clin Psychiatry, 66:17-25.

Harrow M, Yonan CA, Sands JR, et al. 1994. Depression in SchizophreniaAre Neuroleptics, Akinesia, or Anhedonia Involved. Schizophr Bull, 20:327-38.

Hirose S, Ashby CR. 2002. An open pilot study combining risperidone and a selective serotonin reuptake inhibitor as initial antidepressant therapy. J Clin Psychiatry, 63:733-6.

Hirsch SR, Jolley AG. 1989. The dysphoric syndrome in schizophrenia and its implications for relapse. Br J Psychiatry, 155:46-50.

Hirschfeld R, Suppes T, Vieta E, et al. 2006. Quetiapine monotherapy for bipolar II depression: pooled results from two placebo-controlled studies. International Journal of Neuropsychopharmacology, 9:S237.

Hirschfeld RMA, Bowden CL, Gitlin MJ, et al. 2002. Practice Guidelines for the treatment of the patients with Bipolar Disorder (revision). Am J Psychiatry, 159(Suppl 4):1-50.

Hussain MZ, Waheed W, Hussain S, et al. 2005. A comparison of unipolar depression treatment using antidepressants alone versus using antidepressants in combination with quetiapine. Eur Neuropsychopharmacol, 15:S453.

Insel TR. 2006. Beyond efficacy: The STAR*D trial. Am J Psychiatry, 163:5-7.

Jaber M, Robinson SW, Missale C, et al. 1996. Dopamine receptors and brain function. Neuropharmacology, 35:1503-19.

Johnson DAW. 1988. The significance of depression in the prediction of relapse in chronic-schizophrenia. Br J Psychiatry, 152:320-3.

Kapur S, Langlois X, Vinken P, et al. 2002. The differential effects of atypical Antipsychotics on prolactin elevation are explained by their differential blood-brain disposition: A pharmacological analysis in rats. J Pharmacol Exp Ther, 302:1129-34.

Kasper S. 2004. Quetiapine is effective against anxiety and depressive symptoms in long-term treatment of patients with schizophrenia. Depress Anxiety, 20:44-7.

Kasper S, Muller-Spahn F. 2000. Review of quetiapine and its clinical applications in schizophrenia. Expert Opin Pharmacother, 1:783-801.

Keating GM, Robinson DM. 2007. Quetiapine: a review of its use in the treatment of bipolar depression. Drugs, 67:1077-95.

Keck PE Jr, Strakowski SM, McElroy SL. 2000. The efficacy of atypical antipsychotics in the treatment of depressive symptoms, hostility, and suicidality in patients with schizophrenia. J Clin Psychiatry, 61 (Suppl 3):4-9.

Keller MB. 2005. Issues in treatment-resistant depression. J Clin Psychiatry, 66:5-12.

Kennedy SH, Lam RW, Cohen NL, et al. 2001. Clinical guidelines for the treatment of depressive disorders IV. Medications and other biological treatments. Can J Psychiatry, 46:38S-58S.

Khullar A, Chokka P, Fullerton D, et al. 2006. Quetiapine as treatment of non-psychotic unipolar depression with residual symptoms: a double blind, randomized, placebo-controlled study. American Psychiatric Association annual meeting. Toronto, Canada.
Konstantinidis A, Hrubos W, Nirnberger G, et al. 2007. Quetiapine in combination with citalopram in patients with unipolar psychotic depression. Prog Neuropsychopharmacol Biol Psychiatry, 31:242-7.

Lieberman JA, Perkins DO. 2002. Quetiapine: A 5-year update - Introduction. J Clin Psychiatry, 63:3-4.

Martin RL, Cloninger CR, Guze SB, et al. 1985. Frequency and differential-diagnosis of depressive syndromes in schizophrenia. J Clin Psychiatry, 46:9-13.

Mattingly G, Ilivicky H, Canale J, et al. 2006. Quetiapine augmentation for treatment-resistant depression [poster]. The 159th Annual Meeting of the American Psychiatric Association. Toronto, Canada.

McIntyre AW, Gendron A, McIntyre A. 2006. Quetiapine augmentation of SSRIs/SNRIs in major depression with anxiety [poster]. The 159th Annual Meeting of the American Psychiatric Association. Toronto, Canada.

McIntyre RS, Trakas K, Lin D, et al. 2003. Risk of weight gain associated with antipsychotic treatment: results from the Canadian National Outcomes Measurement Study in Schizophrenia. Can J Psychiatry, 48:689-94.

Meltzer HY, Casey DE, Garver DL, et al. 1998. Atypical antipsychotics for treatment of depression in schizophrenia and affective disorders. $J$ Clin Psychiatry, 59:41-5.

Milev R, Abraham G, Zaheer J. 2006. Add-on quetiapine for bipolar depression: a 12-month open-label trial. Can J Psychiatry, 51:523-30.

Muller-Siecheneder F, Muller MJ, Hillert A, et al. 1998. Risperidone versus haloperidol and amitriptyline in the treatment of patients with a combined psychotic and depressive syndrome. J Clin Psychopharmacol, 18:111-20.

Murray CJ, Lopez AD. 1996. The global burden of disease. Boston, Massachusetts: Harvard School of Public Health, World Health Organization, World Bank

Nagy J. 2005. Efficacy, safety and tolerability of quetiapine: short-term high doses with long-term follow-up. Int J Psychiatry Clin Pract, 9:16-21.

Nemeroff CB. 2005. Use of atypical antipsychotics in refractory depression and anxiety. J Clin Psychiatry, 66:13-21.

Nemeroff CB, Gharabawi GM, Canuso CM, et al. 2004. Augmentation with risperidone in chronic resistant depression: A double-blind placebocontrolled maintenance trial. Neuropsychopharmacology, 29:S159.

Nemeroff CB, Kinkead B, Goldstein J. 2002. Quetiapine: preclinical studies, pharmacokinetics, drug interactions, and dosing. J Clin Psychiatry, 63:5-11.

O'Connor M, Silver H. 1998. Adding risperidone to selective serotonin reuptake inhibitor improves chronic depression. J Clin Psychopharmacol, 18:89-91.

Ostroff RB, Nelson JC. 1999. Risperidone augmentation of selective serotonin reuptake inhibitors in major depression. J Clin Psychiatry, 60:256-9.

Papakostas GI, Petersen TJ, Kinrys G, et al. 2005. Aripiprazole augmentation of selective serotonin reuptake inhibitors for treatment-resistant major depressive disorder. J Clin Psychiatry, 66:1326-30.

Papakostas GI, Petersen TJ, Nierenberg AA, et al. 2004. Ziprasidone augmentation of selective serotonin reuptake inhibitors (SSRIs) for SSRIresistant major depressive disorder. J Clin Psychiatry, 65:217-21.

Papakostas GI, Shelton RC, Smith J, et al. 2007. Augmentation of antidepressants with atypical antipsychotic medications for treatmentresistant major depressive disorder: a meta-analysis. J Clin Psychiatry, 68:826-31.

Pathak S, Johns ES, Kowatch RA. 2005. Adjunctive quetiapine for treatmentresistant adolescent major depressive disorder: A case series. $J$ Child Adolesc Psychopharmacol, 15:696-702.

Pitchot W, Ansseau M. 2001. Addition of olanzapine for treatment-resistant depression. Am J Psychiatry, 158:1737-8.

Rubinow DR. 2006. Treatment strategies after SSRI failure - good news and bad news. $N$ Engl J Med, 354:1305-7.

Rush AJ, Trivedi M, Carmody TJ, et al. 2004. One-year clinical outcomes of depressed public sector outpatients: A benchmark for subsequent studies. Biol Psychiatry, 56:46-53. 
Rush AJ, Trivedi MH, Wisniewski SR, et al. 2006. A Comparison of bupropion-SR, sertraline, and venlafaxine-XR following SSRI failure for depressed outpatients: a STAR*D report. $N$ Engl $J$ Med, 354:1231-42.

Sagud M, Mihaljevic-Peles A, Muck-Seler D, et al. 2006. Quetiapine augmentation in treatment-resistant depression: a naturalistic study. Psychopharmacology, 187:511-14.

Sajatovic M, Brescan DW, Perez DE, et al. 2001. Quetiapine alone and added to a mood stabilizer for serious mood disorders. JClin Psychiatry, 62:728-32.

Sajatovic M, Mullen JA, Sweitzer DE. 2002. Efficacy of quetiapine and risperidone against depressive symptoms in outpatients with psychosis. $J$ Clin Psychiatry, 63:1156-63.

Schatzberg A, NemeroffCB. 2006. Essentials of Clinical Psychopharmacology. Arlington, VA: American Psychiatric Publishing Inc.

Seeman P, Tallerico T. 1998. Antipsychotic drugs which elicit little or no Parkinsonism bind more loosely than dopamine to brain D2 receptors, yet occupy high levels of these receptors. Mol Psychiatry, 3:123-34.

Shelton RC, Tollefson GD, Tohen M, et al. 2001. A novel augmentation strategy for treating resistant major depression. Am J Psychiatry, 158:131-4.

Shelton RC, Williamson DJ, Corya SA, et al. 2005. Olanzapine/fluoxetine combination for treatment-resistant depression: a controlled study of SSRI and nortriptyline resistance. J Clin Psychiatry, 66:1289-97.

Simon JS, Nemeroff CB. 2005. Aripiprazole augmentation of antidepressants for the treatment of partially responding and nonresponding patients with major depressive disorder. J Clin Psychiatry, 66:1216-20.

Souery D, Amsterdam J, de Montigny C, et al. 1999. Treatment resistant depression: methodological overview and operational criteria. Eur Neuropsychopharmacol, 9:83-91.

Sprague DA, Loewen PS, Raymond CB. 2004. Selection of atypical antipsychotics for the management of schizophrenia. Ann Pharmacother, 38:313-19.

Tarazi FI, Zhang K, Baldessarini RJ. 2001. Long-term effects of olanzapine, risperidone, and quetiapine on dopamine receptor types in regions of rat brain: Implications for antipsychotic drug treatment. J Pharmacol Exp Ther, 297:711-17.

Tarazi FI, Zhang KH, Baldessarini RJ. 2002. Long-term effects of olanzapine, risperidone, and quetiapine on serotonin $1 \mathrm{~A}, 2 \mathrm{~A}$ and $2 \mathrm{C}$ receptors in rat forebrain regions. Psychopharmacology, 161:263-70.
Targum S, Hassman H, Bastani B, et al. 2005. Use of quetiapine in treating nonpsychotic anxiety and depressive disorder. Schizophr Bull, 31:504-5.

Thase ME, Corya SA, Osuntokun O, et al. 2007. A randomized, doubleblind comparison of olanzapine/fluoxetine combination, olanzapine, and fluoxetine in treatment-resistant major depressive disorder. J Clin Psychiatry, 68: 224-36.

Thase ME, Rush AJ. 1995. Treatment resistant depression. In: Psychopharmacology: the fourth generation of progress. New York, NY: Raven Press.

Thase ME, Macfadden W, Weisler RH, et al. 2006. Efficacy of quetiapine monotherapy in bipolar I and II depression: a double-blind, placebocontrolled study (the BOLDER II study). J Clin Psychopharmacol, 26:600-9.

Tohen M, Vieta E, Calabrese J, et al. 2003. Efficacy of olanzapine and olanzapine-fluoxetine combination in the treatment of bipolar I depression. Arch Gen Psychiatry, 60:1079-88.

Tollefson GD, Sanger TM, Lu YL, et al. 1998. Depressive signs and symptoms in schizophrenia - A prospective blinded trial of olanzapine and haloperidol. Arch Gen Psychiatry, 55:250-8.

Vavrusova L. 2002. Quetiapine in the treatment of non-psychotic depression (P.2 E 037). Int J Neuropsychopharmacol, 5 (Suppl 1).

Vieta E, Reinares M, Corbella B, et al. 2001. Olanzapine as long-term adjunctive therapy in treatment-resistant bipolar disorder. $J$ Clin Psychopharmacol, 21:469-73.

Viner MW, Chen YX, Bakshi I, et al. 2003. Low-dose risperidone augmentation of antidepressants in nonpsychotic depressive disorders with suicidal ideation. J Clin Psychopharmacol, 23:104-6.

World Health Organization. World Health Report 2001. Mental Health: New Understanding, New Hope. Geneva, Switzerland: World Health Organization, 2001.

Yargic LI, Corapcioglu A, Kocabasoglu N, et al. 2004. A prospective randomized single-blind, multicenter trial comparing the efficacy and safety of paroxetine with and without quetiapine therapy in depression associated with anxiety. Int J Psychiatry Clin Pract, 8:205-11.

Yatham LN, Goldstein JM, Vieta E, et al. 2005. Atypical antipsychotics in bipolar depression: Potential mechanisms of action. J Clin Psychiatry, 66:40-8.

Zarate CA, Rothschild A, Fletcher KE, et al. 2000. Clinical predictors of acute response with quetiapine in psychotic mood disorders. $J$ Clin Psychiatry, 61:185-9. 
